\title{
An expectation transformer approach to predicate abstraction and data independence for probabilistic programs*
}

\author{
Ukachukwu Ndukwu and AK McIver \\ Deptartment of Computing, Macquarie University, NSW 2109 Australia. \\ \{ukndukwu, anabel\}@science.mq.edu.au
}

\begin{abstract}
In this paper we revisit the well-known technique of predicate abstraction to characterise performance attributes of system models incorporating probability. We recast the theory using expectation transformers [8], and identify transformer properties which correspond to abstractions that yield nevertheless exact bound on the performance of infinite state probabilistic systems. In addition, we extend the developed technique to the special case of "data independent" programs [14] incorporating probability. Finally, we demonstrate the subtleness of the extended technique by using the PRISM model checking tool [1] to analyse an infinite state protocol, obtaining exact bounds on its performance.
\end{abstract}

\section{Introduction}

Automated analysis of infinite (very large) state systems often relies on abstractions which summarise the essential behaviour as a finite state "anti-refinement" in such a way as to guarantee the desired properties (if indeed they hold). Typically, however, abstractions introduce nondeterminism, and in a probabilistic system this can lead to a high degree of imprecision in the estimated probabilistic properties. The choice of abstraction therefore is critical; some approaches to finding the right one use "abstraction refinement", sometimes relying on counterexamples of failed attempts to obtain incremental improvements [7].

In this paper we revisit the technique of "predicate abstraction" from the perspective of "expectation transformers". Predicate abstraction refers to the notion of approximating a system using a given set of predicates: states are grouped together according to the predicates they satisfy (in the given set), and the system is abstracted by tracking only the transformations expressible in the induced equivalence classes. Expectation transformers [8] is a generalisation to probabilistic systems of Hoare/Dijkstra-style semantic reasoning [20] - predicates are replaced by real-valued functions of the state. The approach is equivalent to operational models of programming based on Markov-Decision Processes, but results in a convenient proof system for verifying general properties of probabilistic programs.

In particular we are able to characterise, using expectation transformers, a simple criterion for when an abstraction gives exact quantitative analysis for probabilistic properties. The criterion is sufficient to identify when predicate abstraction introduces no additional nondeterminism. A typical class of programs where this is effective is the so-called class of "data independent" programs [14]. A program is data independent whenever its control structure does not depend on the precise values of the data. Wolper [14] first identified this as a class of interesting programs amenable to verification via model checking [9]. In addition to Wolper's idea, we consider the notion of probabilistic data independence where the probabilistic choice cannot be dependent on the data. In general, the idea we propose here is aimed

${ }^{*}$ The authors acknowledge support from (I) The Australian Commonwealth Endeavour International Postgraduate Research Scholarship (E-IPRS) Fund, and (II) The Australian Research Council (ARC) Grant Number DP0879529.

A. Di Pierro \& G. Norman (Eds): 8th Workshop on Quantitative Aspects of Programming Languages (QAPL 2010)

EPTCS 28, 2010, pp. 129-143 doi 10.4204/EPTCS.28.9 (c) Uk Ndukwu and AK McIver

This work is licensed under the Creative Commons Attribution License. 
at constructing abstractions which result in no loss of information especially when probability plays a crucial role in the performance analysis of infinite state system models. Such abstractions are said to be "information-preserving" since they suffice as exact representations of their original systems.

Using the expectation transformer approach we prove the "folk theorem" (see [4]) for probabilistic systems: that data independent programs can be treated with predicate abstraction yielding exact results on threshold properties such as "the probability that a set of states has been reached in at most $k$ steps".

In particular our contributions in this paper are:

(i) The development of a technique which permits the application of predicate abstraction to probabilistic programs using expectation transformers;

(ii) An establishment of a criterion for identifying abstractions which do not lose information;

(iii) We show how the developed technique and criterion can be applied to data independent programs especially when probability plays a crucial role;

(iv) And finally, a demonstration of the technique on a case study of a system with potential infinite state behaviour.

This paper is structured as follows: In Sec. 2 we summarise the expectation transformer semantics for probabilistic programs, Sec. 3 is the development of the technique for predicate abstraction using expectation transformers. In Sec. 4 we show how the technique can be applied to identify when predicate abstraction yields exact thresholds for infinite state systems; we then explore the special treatment of data independent programs. In Sec. 5] we illustrate the technique by model checking the Rabin's choice coordination problem (also known as the distributed consensus) [13]; this is a protocol which has the potential to require unbounded resources on its performance and therefore cannot be verified directly with a model-checking approach. However the theory of Sec. 4 1 shows that the results we obtain using its information-preserving abstraction are nevertheless exact interpretations of its performance.

\subsection{Summary of notation}

Function application is represented by a dot, as in $f \cdot x$ (rather than $f(x)$ ). We use an abstract state space $S$. Given predicate Pred we write $[$ Pred $]$ for the characteristic function mapping states satisfying Pred to 1 and to 0 otherwise, punning 1 and 0 with "True" and "False" respectively. Whenever $e, e^{\prime}$ are real-valued functions over $S$ we write $e+e^{\prime}, e \sqcup e^{\prime}, e \sqcap e^{\prime}$ for the pointwise addition, maximum and minimum. Moreover $\alpha \times e$ is $e$ scaled by the real $\alpha$.

For commutative operator $\odot$, we use $(\odot x \in X \cdot f \cdot x)$ for the comprehension which applies $\odot$ between all instances $f \cdot x$ as $x$ ranges over $X$. For example, $\left(\sqcup x \in[0,1] \cdot x^{2}\right)$ gives the maximum value of $x^{2}$ as $x$ ranges over the closed interval $[0,1]$.

\section{Probabilistic program semantics and expectation transformers}

When programs incorporate probability, their properties can no longer be guaranteed "with certainty", but only "up to some probability". For example the program

$$
\text { inc } \hat{=} x:=x / 2_{1 / 2} \oplus x+1
$$

sets the integer-valued variable $x$ to $x / 2$ (the result of the integer division) only with probability $1 / 2$

- in practice this means that if the statement (1) were executed a large number of times, and the number 
of times that $x$ was halved or increased tabulated, roughly $1 / 2$ of them would record $x$ as having been halved (up to well-known statistical confidence [15]).

The probabilistic guarded command language pGCL [8] and its associated quantitative logic were developed to express such programs and to derive their probabilistic properties by extending the classical assertional style of programming. Programs in the pGCL are modeled (operationally) as functions (or transitions) which map initial states in $S$ to (sets of) probability distributions over final states - the program at (11) for instance has a single transition which maps any initial state $x=k_{0}$ to a (single) final distribution; we represent that distribution as a function $d$, evaluating to $1 / 2$ when $x=k_{0} / 2$ or $x=k_{0}+1$.

Since properties are now quantitative we express them via a logic of real-valued functions, or expectations. For example, the property "if the initial state satisfies $x=0 \vee x=2$, then the final value of $x$ is 1 with probability $1 / 2$ " can be expressed as the expected value of the function $[x=1]$ with respect to $d$, which evaluates to $1 / 2 \times 1+1 / 2 \times 0=1 / 2$, when $x$ is initially 2 for example.

Direct appeal to the operational semantics quickly becomes impractical for all but the simplest programs - better is the equivalent transformer-style semantics which is obtained by rationalising the above calculation in terms of expected values rather than transitions, and the explanation runs as follows. Writing $\mathscr{E} S$ for the set of all (non-normalised) functions from $S$ to the interval $[0,1]$, which we call the set of expectations, we say that the expectation $[x=1]$ has been transformed to the expectation $[x=0 \vee x=2] / 2$ by the program inc (11) above so that they are in the relation " $1 / 2$ is the expected value of $[x=1]$ with respect to inc's result distribution whenever $x$ is initially either 0 or 2". More generally given a program Prog, an expectation $e$ in $\mathscr{E} S$ and a state $s \in S$, we define wp.Prog.e.s to be the expected value of $e$ with respect to the result distribution of program Prog if executed initially from state $s$. We say that wp.Prog is the expectation transformer relative to Prog. In our example that allows us to write

$$
[x=0 \vee x=2] / 2=\operatorname{wp} \cdot\left(x:=x / 2_{1 / 2} \oplus x:=x+1\right) \cdot[x=1] .
$$

In the case that nondeterminism is present, execution of Prog results in a set of possible distributions and we modify the definition of wp to take account of this - indeed we define wp.Prog.E.s so that it delivers the least-expected value with respect to all distributions in the result set. The transformers [8] give rise to a complete characterisation of probabilistic programs with nondeterminism, and they are sufficient to express many performance-style properties, including the probability that an event occurs, the expected time that it occurs, and long-run average of the number of times it occurs over many repeated executions of the system.

In Fig. 1 we set out the semantics for the pGCL, a variation of Dijkstra's GCL with the addition of probabilistic choice. All the programming features have been defined previously elsewhere, and (apart from probabilistic choice) have interpretations which are merely adapted to the real-valued context. For example, nondeterminism, as explained above, is interpreted demonically and can be thought of as being resolved by a "minimal-seeking demon", providing guarantees on all program behaviour, such as is expected for total correctness. Probabilistic choice, on the other hand, selects the operands at random with weightings determined by the probability parameter $p$. Iteration is defined by a least fixed point of a monotone expectation-to-expectation function 1

We end this section with a discussion of a simple performance property: a probabilistic analysis of the number of iterations until termination. Given a loop do $G \rightarrow$ Prog od which executes the program Prog until $G$ becomes false, we can compute the probability that the loop has executed no more than $k$

\footnotetext{
${ }^{1}$ Well-definedness is guaranteed by, for example, restricting the expectations to lie in the real interval $[0,1]$ or to complete the reals with $\infty$. These issues have been discussed elsewhere [8].
} 


$\begin{array}{ll}\text { skip } & \text { wp.skip. } E \hat{=} E, \\ \text { abort } & \text { wp.abort. } E \hat{=} 0, \\ \text { assignment } & \text { wp. }(x:=f) \cdot E \hat{=} E[x:=f], \\ \text { sequential composition } & \text { wp. }\left(r_{9}^{\circ} r^{\prime}\right) \cdot E \hat{=} \text { wp.r. }\left(\text { wp. } r^{\prime} . E\right), \\ \text { probabilistic choice } & \text { wp. }\left(r_{p} \oplus r^{\prime}\right) \cdot E \hat{=} p \times \text { wp.r. } E+(1-p) \times \text { wp. } r^{\prime} . E, \\ \text { nondeterministic choice } & \text { wp. }\left(r \llbracket r^{\prime}\right) \cdot E \hat{=} \text { wp.r. } \sqcap \text { wp. } r^{\prime} \cdot E, \\ \text { Boolean choice } & \left.\text { wp. (if } G \text { then } r \text { else } r^{\prime}\right) \cdot E \hat{=}[G] \times \text { wp.r. } E+[\neg G] \times \text { wp. } r^{\prime} . E, \\ \text { Iteration } & \text { wp.(do } G \rightarrow r \text { od }) \cdot E \hat{=}(\mu X \bullet[\neg G] \times E+[G] \times \text { wp.r.X). }\end{array}$

$E$ is an expectation in $\mathscr{E} S$, and $f$ is a function of the state, and $\square$ is pointwise minimum. The real $p$ is restricted to lie between 0 and 1 , and the term $(\mu X \ldots)$ refers to the least fixed point with respect to $\leq$, which we lift to realvalued functions. Commands are ordered using refinement, so that more refined programs improve probabilistic results, thus $P \sqsubseteq Q$ iff ( $\forall E \in \mathscr{E} S \cdot$ wp.P.E $\leq$ wp.Q.E); note also that the monotone property of wp is such that if $E \leq E^{\prime}$ then wp.P.E $\leq$ wp.P. $E^{\prime}$, where $P, Q$ are program commands and $E, E^{\prime}$ are expectations.

Figure 1: Structural definitions of wp for the pGCL.

times on termination as:

$$
\text { wp.do } G \rightarrow \operatorname{Prog} ; n:=n+1 \text { od. }[n \leq k] \text {, }
$$

where $n$ is a fresh variable, not occurring in Prog. Informally, if $n$ is initialised to 0 before the execution of the loop and is incremented after each execution of Prog, this expresses the (minimum) probability that its value on exiting the loop does not exceed $k$. When no nondeterminism is present the expression in (2) computes an exact bound for expected performance; when it is present it computes the greatest lower bound. However upper bounds can be calculated using a maximum interpretation of nondeterminism but we do not discuss that interpretation here.

In this section we have summarised an expectation transformer approach to probabilistic semantics. In many cases, especially for performance, the exact analysis of the system in this style is impractical; an alternative to calculation is model checking, however this is not viable for very large or infinite systems. Predicate abstraction is a popular approach to approximating such programs, and in the next section we develop the expectation transformer approach to predicate abstraction for probabilistic programs.

\section{Abstract expectation transformers}

Predicate abstraction is a standard technique for defining abstractions of transition systems. In this section we will show how to define it for probabilistic programs using expectation transformers. The approach is inspired by Ball's formalisation of predicate abstraction for standard sequential programs using weakest precondition semantics [17].

Let $\Phi$ be a (finite) set of predicates over the state space $S$. The standard predicate abstraction over $\Phi$ is induced by the equivalence class:

$$
s \sim_{\Phi} s^{\prime} \quad \text { iff } \quad\left(\forall \phi \in \Phi \cdot \phi . s=\phi . s^{\prime}\right) .
$$

Given a transition system $T$ over $S$, the abstract transition system $T / \sim_{\Phi}$ takes the equivalence classes given by $S / \sim_{\Phi}$ as the state space, and their transitions $\hat{s} \rightarrow \hat{t}$ in $T / \sim_{\Phi}$ provided that there exists a 
transition $s \rightarrow t$ in $T$. The probabilistic generalisation is somewhat more complicated to define. On the other hand the expectation transformer semantics characterises operational behaviour, and the approach we take here is to define the abstract transition system over $S / \Phi$ using a generalisation of Ball's idea.

Let cubes. $\Phi$ be the (finite) set of (non trivial) minimal predicates formed by taking negations and conjunctions of predicates in $\Phi$. The set cubes. $\Phi$ corresponds to the (set of) equivalence classes $S / \sim_{\Phi}$, and represents the abstract state space of the abstraction induced by $\Phi$. Let cubed $: \mathscr{E} S \rightarrow \mathscr{E} S$ be defined

$$
\text { cubed }_{\Phi} . e \hat{=}(\sqcup c \in \text { cubes. } \Phi \cdot(\sqcup \lambda[c] \leq e \cdot \lambda[c])) .
$$

We note that $\operatorname{cubed}_{\Phi} . e$ is unique and would usually be a linear combination of the elements of cubes. $\Phi$, hence making it the sum of scaled cubes over the latter. Consequently, cubed.e is the weakest approximation of $e$ with respect to the granularity expressible by conjunctions, negations and disjunctions in $\Phi$. We say that $e$ is $c u b e d$ relative to $\Phi$ exactly when $e=\operatorname{cubed}_{\Phi} . e$. Note that cubed.cubed.e $=$ cubed. $e$ and that sums, maxima and minima of cubed expressions are still cubes, i.e.

$$
\begin{aligned}
& 3(a) \quad \text { cubed. }\left(\text { cubed. } e+\text { cubed. } e^{\prime}\right)=\left(\text { cubed. } e+\text { cubed. } e^{\prime}\right), \\
& 3(b) \quad \text { cubed. }\left(\text { cubed. } e \sqcup \text { cubed. } e^{\prime}\right)=\left(\text { cubed. } e \sqcup \text { cubed. } e^{\prime}\right), \\
& 3(c) \quad \text { cubed. }\left(\text { cubed.e } \sqcap \text { cubed. } e^{\prime}\right)=\left(\text { cubed.e } \sqcap \text { cubed. } e^{\prime}\right) .
\end{aligned}
$$

Definition 1. Given a pGCL program Prog, and a set of predicates $\Phi$, and expectation e over $S$ we define the abstract weakest expectation relative to $\Phi$ as:

$$
\mathrm{wp}_{\Phi} . \text { Prog.e } \hat{=} \text { cubed }_{\Phi} . \mathrm{wp} . \text { Prog.e. }
$$

We write $\operatorname{Prog}_{\Phi}$ for the corresponding abstract program operating over the abstract system $S / \Phi$. This implies that Prog $\operatorname{P}_{\Phi}$ is determined by $\mathrm{wp}_{\Phi}$. Prog.

As an example, consider the program inc at (1) operating under arithmetic modulo 4 . The underlying state space is defined by $0 \leq x<4$; consider now the set $\Phi$ consisting of the single predicate $x=0 \vee x=2$; the set of cubes cubes. $\Phi \hat{=}\{(x=0 \vee x=2),(x=1 \vee x=3)\}$, implying that the induced predicate abstraction has two states. We can see now that

$$
\begin{aligned}
\quad \mathrm{wp}_{\Phi} \cdot \text { inc } \cdot[x=1 \vee x=3] & =[x=0 \vee x=2] / 2, \\
\text { and } \quad \mathrm{wp}_{\Phi} \cdot \operatorname{inc} \cdot[x=0 \vee x=2] & =[x=1 \vee x=3] / 2,
\end{aligned}
$$

which is consistent with the abstraction in Fig. 2. where each abstract state has a probability of at least $1 / 2$ of being transformed to the other state, with the remaining probability being assigned to a nondeterministic update.

The next lemma sets out some properties of the abstract expectation transformer.

Lemma 1. Let Prog, Prog' be programs, $\Phi, \Phi^{\prime}$ sets of predicates, and e, $e^{\prime}$ expectations and $\alpha$ a real. The following inequalities apply:

$$
\begin{aligned}
& \mathrm{wp}_{\Phi} \cdot \text { Prog.e } \\
& \mathrm{wp}_{\Phi} \cdot \text { Prog.e } \\
& \mathrm{wp}_{\Phi} \cdot \text { Prog.e }+\mathrm{wp}_{\Phi} \cdot \text { Prog.e } \\
& \alpha \times \mathrm{wp}_{\Phi} \cdot \text { Prog.e } \\
& \left(\mathrm{wp}_{\Phi} \cdot \text { Prog.e-1) } \sqcup 0\right)
\end{aligned}
$$

$\leq \quad$ wp.Prog.e
$\leq \quad \mathrm{wp}_{\Phi \cup \Phi^{\prime} \cdot \text { Prog.e }}$
$\leq \quad \mathrm{wp}_{\Phi} \cdot$ Prog. $\left(e+e^{\prime}\right)$
$=\quad \mathrm{wp}_{\Phi} \cdot \operatorname{Prog} \cdot(\alpha \times e)$
$\leq \quad \mathrm{wp}_{\Phi} \cdot \operatorname{Prog} \cdot((e-1) \sqcup 0)$

Proof: The inequalities and equalities all follow from arithmetic and Def. [1 


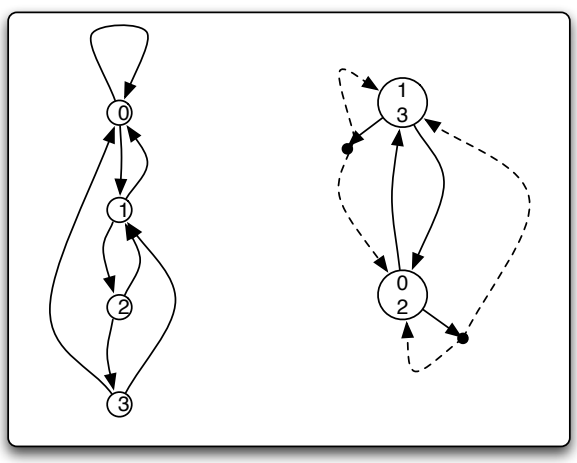

The transition system on the left represents the program inc over the state space defined by $0 \leq x<4$, using arithmetic modulo 4 . Each solid black arrow occurs with probability $1 / 2$. The transition system on the right is the abstraction based on $\Phi=\{x=0 \vee x=2\}$. Here we can see non determinism (indicated by dotted lines) is introduced after any transition which divides the value of $x$ by 2 .

Figure 2: The transition system for inc and an abstraction.

Lem. 1 confirms our intuition that (1) the properties measured with respect to the abstraction are no more than with respect to the original program; (2) finer-grained abstractions give more accurate results and $(3,4,5) w p_{\Phi}$.Prog corresponds to a well-defined probabilistic transition system [8].

For standard transitions systems (with no probability) an abstract system $\operatorname{Prog}_{\Phi}$ is determined directly from the control structure and assignment statements. This corresponds to $\mathrm{wp}_{\Phi}$ distributing through the program operators. For probabilistic systems this is not the case. For example, the program inc; inc

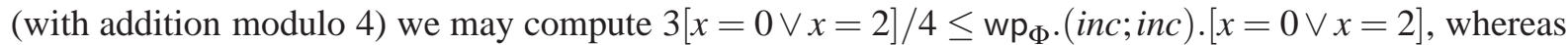
$[x=0 \vee x=2] / 4=\mathrm{wp}_{\Phi} \cdot i n c \cdot\left(\mathrm{wp}_{\Phi} \cdot i n c\right) \cdot[x=0 \vee x=2]$, implying that the nondeterminism introduced at each abstract transition will increase the inaccuracy. Comparing with Fig. 2 we see that nondeterminism is introduced at each abstract transition, and this could be resolved in the abstract system in such a way that there is only $1 / 4$ chance of returning to the initial abstract state.

The following lemma shows that $\mathrm{wp}_{\Phi}$ only distributes through nondeterminism, and only subdistributes through sequential composition and probabilistic choice.

Lemma 2. Let Prog, Prog' be programs, $\Phi, \Phi^{\prime}$ sets of predicates, and e, $e^{\prime}$ expectations. The following inequalities apply:

$$
\begin{aligned}
& \mathrm{wp}_{\Phi} \cdot\left(\operatorname{Prog} \llbracket \operatorname{Prog}^{\prime}\right) . e \quad=\quad \mathrm{wp}_{\Phi} \text {.Prog.e } \sqcap \mathrm{wp}_{\Phi} \cdot \text { Prog }^{\prime} . e \\
& \mathrm{wp}_{\Phi} \cdot \text { Prog. }\left(\mathrm{wp}_{\Phi} \cdot \text { Prog }^{\prime}\right) \cdot e \leq \mathrm{wp}_{\Phi} \cdot\left(\text { Prog}_{\text {Prog } \left.^{\prime}\right) \cdot e}\right. \\
& \mathrm{wp}_{\Phi} \cdot\left(\operatorname{Prog}_{p} \oplus \mathrm{wp}_{\Phi} \cdot \operatorname{Prog}^{\prime} \cdot e \leq \mathrm{wp}_{\Phi} \cdot\left(\operatorname{Prog}_{p} \oplus \operatorname{Prog}^{\prime}\right) \cdot e\right.
\end{aligned}
$$

Proof: The inequalities and equalities all follow from arithmetic and Def. [1]

Lem. 2 implies that whenever nondeterminism is introduced, the analysis of a program abstracted at each program statement could be too coarse to verify a desired quantitative threshold. This is not a problem when the abstraction does not introduce nondeterminism. Consider the program

$$
\text { twoFlip } \hat{=} x:=0_{p} \oplus x:=1 ; y:=0_{q} \oplus y:=1,
$$

and the set of predicates $\Phi \hat{=}\{x=y, x \neq y\}$. The resulting transition system over the state space defined by $x$ and $y$ is set out in Fig. 3 together with the abstraction induced by this $\Phi$. 


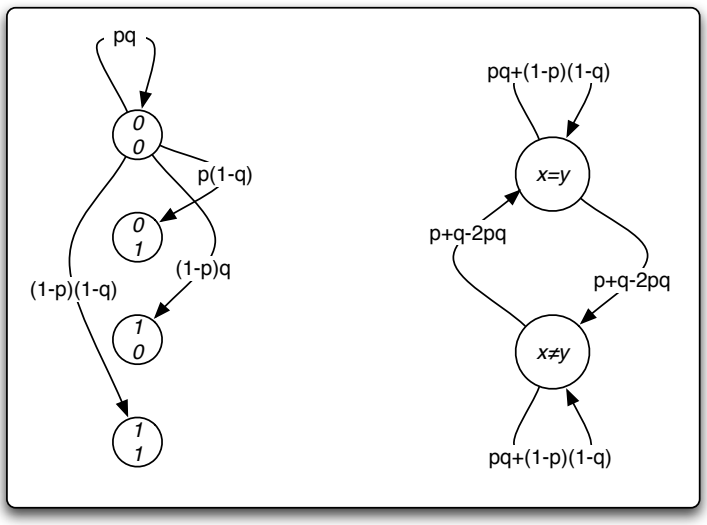

The transition system (labelled with probabilities) on the left represents the program twoFlip over the state space defined by variables $x$ and $y$, each of which can take 0 or 1 value in the states $(x, y)$. Each branch is executed with the probability that it occurs; only the transitions from $x=y=0$ are illustrated, with transitions from the remaining states similarly calculated. The transition system on the right represents the abstraction which only keeps track of whether $x$ and $y$ are equal or not. Since no nondeterminism is introduced, properties at that level of granularity can be accurately calculated using this abstraction.

Figure 3: The transition system for twoFlip and an abstraction.

Observe how no nondeterminism has been introduced in this abstraction — since indeed $w_{\Phi} \cdot($ twoFlip; twoFlip $)=w_{\Phi} \cdot($ twoFlip $) ; w_{\Phi} \cdot(t w o F l i p)$. Intuitively this tells us that properties which can be stated at the granularity of $\Phi$ can be computed accurately from its corresponding abstraction. In the next section we formalise our intuition using expectation transformers.

\section{Information-preserving abstractions and expected time to terminate}

In this section we introduce "information-preserving" abstractions and study how they apply to the computation of exact bounds on performance-style properties of probabilistic programs.

As we saw above, an abstraction which does not introduce nondeterminism preserves the exact behaviour of the program at the granularity of the chosen set of predicates. Programs which do not exhibit nondeterminism or aborting behaviours satisfy the special properties that:

$$
\begin{aligned}
& \mathrm{wp}_{\Phi} \cdot \text { Prog. }\left(e+e^{\prime}\right)=\mathrm{wp}_{\Phi} \cdot \text { Prog.e }+\mathrm{wp}_{\Phi} \cdot \text { Prog.e } \\
& \mathrm{wp}_{\Phi} \cdot \text { Prog. }(1-e)=1-\mathrm{wp}_{\Phi} \text {.Prog.e }
\end{aligned}
$$

for any deterministic pGCL program command Prog, set of predicate $\Phi$, and expectations $e, e^{\prime}$. The next definition formalises that idea in terms of expectation transformers.

Definition 2. Given a deterministic program Prog and a set of predicates $\Phi$, we say that the abstraction induced by $\Phi$ is information-preserving if:

$$
\mathrm{wp}_{\Phi} \cdot \operatorname{Prog} \cdot[c]=\text { wp.Prog. }[c] \text {, }
$$

for all $c \in$ cubes. $\Phi$.

To see Def.2 in action, observe that 


$$
\begin{array}{ll} 
& \text { wp.inc. }[x=0 \vee x=2] \\
= & {[x=0 \vee x=3] / 2+[x=1]} \\
\neq \quad & {[x=1 \vee x=3] / 2} \\
=\quad & \operatorname{wp}_{\Phi} \cdot \text { inc. }[x=0 \vee x=2],
\end{array}
$$

implying the introduction of a nondeterministic branch at the abstract state corresponding to $x=1 \vee x=3$.

A more efficient way to check for information-preservation is simply to check that wp.Prog. $[\phi]$ is cubed for all $\phi \in \Phi$; the next lemma shows that this is sound.

Lemma 3. Let Prog be a deterministic (probabilistic) program, and let $\Phi$ be a set of predicates. If wp.Prog. $[\phi]$ is cubed for all $\phi \in \Phi$ then the abstraction induced by $\Phi$ is information-preserving.

Proof: We need to show that wp.Prog. $[c]=\mathrm{wp}_{\Phi}$.Prog. $[c]$ for all $c \in$ cubed. $\Phi$. Note that each such $c$ is generated from negations and conjunctions, so all we need show is that for predicates $\psi, \psi^{\prime}$ such that wp.Prog. $[\psi]$ and wp.Prog. $\left[\psi^{\prime}\right]$ are cubed, then so too are wp.Prog. $(1-[\psi])$ and wp.Prog. $\left[\psi \wedge \psi^{\prime}\right]$.

The result follows since wp.Prog. $\left[\psi \wedge \psi^{\prime}\right]=$ (wp.Prog. $[\psi]+$ wp.Prog. $\left.[\psi]^{\prime}-1\right) \sqcup 0$, and wp.Prog. $(1-$ $[\psi])=1-$ wp.Prog. $[\psi]$, and the fact that sums and inverses of cubed expressions are still cubed.

As mentioned above, a key characterising property of information-preserving abstractions is that they generate no new nondeterminism. A probabilistic program exhibits no (demonic) nondeterminism if its expectation transformer semantics distributes addition. The next lemma shows this for informationpreserving abstractions.

Lemma 4. Let Prog be a deterministic (probabilistic) program, and let $\Phi$ be a set of predicates inducing an information-preserving abstraction on Prog. The predicate transformer $\mathrm{wp}_{\Phi}$.Prog is deterministic on cubed expectations.

Proof: The result follows by showing that $\mathrm{wp}$. Prog $=\mathrm{wp}_{\Phi}$.Prog on cubed expressions. Assume first that $c, c^{\prime} \in$ cubes. $\Phi$, and that $\lambda, \lambda^{\prime}$ are reals. We reason as follows:

$$
\begin{aligned}
& \mathrm{wp}_{\Phi} \cdot \operatorname{Prog} \cdot\left(\lambda[c]+\lambda^{\prime}\left[c^{\prime}\right]\right) \\
& \leq \quad \quad \text { wp.Prog. }\left(\lambda[c]+\lambda^{\prime}\left[c^{\prime}\right]\right) \\
& =\quad \lambda \text { wp.Prog. }[c]+\lambda^{\prime} \text { wp.Prog. }\left[c^{\prime}\right] \\
& =\quad \lambda \mathrm{wp}_{\Phi} \cdot \operatorname{Prog} \cdot[c]+\lambda^{\prime} \mathrm{wp}_{\Phi} \cdot \operatorname{Prog} \cdot\left[c^{\prime}\right] \\
& \leq \quad w_{\Phi} \cdot \operatorname{Prog} \cdot\left(\lambda[c]+\lambda^{\prime}\left[c^{\prime}\right]\right) .
\end{aligned}
$$

Prog is deterministic

Prog is information-preserving

Lem. प(3)

Observe finally that the equality generalises for expressions consisting of finite sums of cubes, and the fact that there are only finitely many distinct cubes whenever $\Phi$ is finite.

In particular we can now see that information-preserving abstractions compute exact results for all cubed expressions:

Corollary 1. Let Prog be a deterministic (probabilistic) program, and let $\Phi$ be a set of predicates inducing an information-preserving abstraction on Prog. Then $\mathrm{wp}_{\Phi}$. Prog.e $=\mathrm{wp}$.Prog.e whenever e is cubed. Proof: Follows since if $e$ is cubed then it is a finite sum of scaled cubes, and by Lem. $4 \mathrm{wp}_{\Phi}$. Prog distributes addition.

\subsection{Computing abstractions component-wise}

The above notions assume that the abstraction is calculated wholesale on the program Prog; in practice it may be more efficient to calculate the abstraction by computing it relative to, and on smaller components of the program, however as Lem. 2 (5,6) indicate, additional inaccuracies can creep in wherever the abstraction is computed from program components. 
Fortunately this does not occur in the case of information-preserving abstractions: Lem. 4 is key to verifying that information-preserving abstractions are determined from their components alone, provided that they themselves are also information-preserving. In practical terms this means that in a transitionsystem, provided each transition preserves the information, then so will the abstraction. In our predicate transformer framework, we need to show that $\mathrm{wp}_{\Phi}$ distributes sequential composition and probabilistic choice.

Lemma 5. Let Prog, Prog' be deterministic (probabilistic) programs, and let $\Phi$ be a set of predicates inducing an information-preserving abstraction on each. The following inequalities apply:

$$
\begin{aligned}
& \mathrm{wp}_{\Phi} \cdot \operatorname{Prog} \cdot\left(\mathrm{wp}_{\Phi} \cdot \operatorname{Prog}^{\prime}\right)=\mathrm{wp}_{\Phi} \cdot\left(\operatorname{Prog}_{\boldsymbol{P}} \operatorname{Prog}^{\prime}\right) \\
& \mathrm{wp}_{\Phi} \cdot \operatorname{Prog}_{p} \oplus \mathrm{wp}_{\Phi} \cdot \operatorname{Prog}^{\prime}=\mathrm{wp}_{\Phi} \cdot\left(\operatorname{Prog}_{p} \oplus \operatorname{Prog}^{\prime}\right) .
\end{aligned}
$$

Proof: Follows easily from Lem. 4 4 since $w_{\Phi}$. Prog and $w p_{\Phi} \cdot$ Prog' are both cubed expressions.

\subsection{Computing average performance}

Significantly, we can now compute expected performance profiles exactly from the abstraction.

Lemma 6. Let Prog be a deterministic program, and information-preserving with respect to $\Phi$, and suppose that $G,(n \leq k) \in \Phi$, where $k$ is an integer. The following equalities hold:

$$
\text { wp. (do } G \rightarrow \operatorname{Prog} ; n:=n+1 \text { od }) .[n \leq k]=\text { wp. }\left(\text { do } \hat{G} \rightarrow \operatorname{Prog}_{\Phi} ; n:=n+1 \text { od }\right) .[n \leq k] \text {, }
$$

where $\hat{G}$ represents the abstraction of $G$ in $S / \sim_{\Phi}$.

Proof:

Let $N \hat{=}$ wp.(do $G \rightarrow \operatorname{Prog} ; n:=n+1$ od). $[n \leq k]$, and $N_{\Phi} \hat{=}$ wp.(do $\hat{G} \rightarrow \operatorname{Prog}_{\Phi} ; n:=n+1$ od). $[n \leq k]$. By Lem. 1 (1), and monotonicity of the programming language Fig. 1 we see that $N_{\Phi} \leq N$. To show that $N \leq N_{\Phi}$ we note that $N$ and $N_{\Phi}$ are both least fixed points of monotone expectation-to-expectation functions. We use the least fixed point property of functions over partially-ordered sets, i.e. that if $f . x \leq x$ then $\mu . f \leq x[2]$. Applied to $N$ and $N_{\Phi}$ we establish that $N_{\Phi}$ satisfies the least fixed point equation for $N$ as follows:

$$
\begin{array}{llr} 
& {[G] \times[n \leq k]+[\neg G] \times \mathrm{wp}_{\Phi} \cdot \text { Prog. } N} & \\
= & {[G] \times[n \leq k]+[\neg G] \times \text { wp.Prog. } N_{\Phi}} & \operatorname{cubed}_{\Phi} . N_{\Phi}=N_{\Phi} \text { (see below); Lem. } \text { (4) } \\
= & N_{\Phi} . & N_{\Phi} \text { is a fixed point }
\end{array}
$$

The result now follows since $N$ is the least fixed point of the function $(\lambda x \cdot[G] \times n+[\neg G] \times$ wp.Prog.x).

For the "see below" part, note that $N_{\Phi}$ is itself a fixed point, satisfying: $N_{\Phi}=[G] \times[n \leq k]+$ $[\neg G] \times \mathrm{wp}_{\Phi}$.Prog. $N_{\Phi}$. It now follows that $N_{\Phi}$ is cubed since $\mathrm{wp}_{\Phi}$.Prog.e is, for any expression e.

More generally exact bounds can be computed even when the program exhibits finitely-branching nondeterminism.

Corollary 2. Let Prog $_{1} \ldots$ Prog $_{m}$ be deterministic and information-preserving with respect to $\Phi$. Let $G \in \Phi$, and $n$ a fresh variable. The following equality holds:

$$
\begin{aligned}
& \text { wp.Prog. }\left(\text { do } G \rightarrow\left(\operatorname{Prog}_{1} \rrbracket \ldots \rrbracket \operatorname{Prog}_{m} \text { od }\right) \cdot[n \leq k]\right. \\
= & \text { wp.Prog.(do } G \rightarrow\left(\operatorname{Prog}_{1 \Phi} \rrbracket \ldots \rrbracket \operatorname{Prog}_{m \Phi} \text { od }\right) \cdot[n \leq k] .
\end{aligned}
$$

Proof: $\quad$ The proof is similar to Lem. [ since nondeterminism distributes by Lem. [1 (4).

The significance of Cor. 2 is that whenever the abstraction is known to be information-preserving component-wise over a program (or transition system), then exact performance can be carried out on the abstraction. An important class of such programs are the so-called "data independent" systems, to which we turn in the next section. 


\subsection{Data independence}

A program is said to be data independent (with respect to a data type $X$ ) [14] if it cannot perform operations involving specific values of the type: specifically it can only input, output, store and make comparisons using any relational operator $\Theta \in\{=,<, \leq,>, \geq, \ldots\}$. Wolper points out that many distributed protocols fall into this category - he shows that such systems can be model checked accurately. In fact, if we extend this informal definition to probabilistic programs such that all probabilistic choices are constants, then our results above imply that there is an abstraction which can be used to compute performance properties exactly, namely the abstraction induced by predicates $\Psi \hat{=}\{x \Theta y \mid \forall x, y$ program variables of same type $\}$. We use this intuition to define a simple characterisation of data independent programs: they are the programs which are information-preserving with respect to $\Psi$ (with informal definition above).

Definition 3. Let Prog be a deterministic pGCL program with variables $x_{1} \ldots x_{m}$. We say that Prog is data independent with respect to $x_{1} \ldots x_{m}$ provided that Prog is information-preserving with respect to the abstraction induced by $\Psi$, where $\Psi$ is the set of predicates containing all expressions of the form $x_{i} \Theta y_{j}$ for all $1 \leq i, j, \leq m$.

Note that this characterisation of data independence can be generalised to programs $\operatorname{Prog}_{1} \llbracket \ldots \square \operatorname{Prog}_{n}$ which exhibit nondeterministic behaviour by ensuring that the deterministic components Prog $_{i}$ comply with Def.3 Note that this definition shares some similarities with Wolper's denotational characterisation [14], in that Def. 3 captures the idea that properties expressible at the granularity of $\Phi$ are shared by both $\operatorname{Prog}_{\Phi}$ and Prog. It does not deal with general types however, as does Lazic [16].

With Def. 3 we can now conclude that data independent probabilistic programs have abstractions which preserve performance bounds.

Lemma 7. Let Prog be a data independent program. Then the expected number of iterations do $G \rightarrow$ Prog od may be computed exactly using the abstract program $\operatorname{Prog}_{\Psi}$ whenever $G \in \Psi$, where $\Psi$ is defined in Def. 3 .

The practical implication of Lem. 7(which follows directly from Lem. 6 and Cor. 2) is that performance (and correctness) of data independent programs can be analysed exactly using model checking. In the next section we give an example to illustrate this idea.

\section{Case study: Rabin's distributed consensus}

We illustrate the effectiveness of our technique on the Rabin's choice-coordination problem [13]. The state space generated on execution of the algorithm is potentially infinite hence limiting the scope of model checking on verifying liveness properties (such as termination conditions) relating to its overall performance. As we will see, even though the algorithm is not quite data independent, there does exist an information-preserving abstraction demonstrating that exact numerical analysis is still possible on its performance.

\subsection{Informal description}

A group of tourists are to decide between two meeting places (which are not of interest to us). A major constraint is that they may not communicate as a group; nor is there a central "authority" (e.g. a tour guide) whose decision overrides theirs. 
Each tourist carries a notepad on which he will write various numbers; outside each of the two potential meeting places is a noticeboard on which various messages will be written. Initially the number zero appears on all the notepads and on the two noticeboards.

Each tourist decides independently (demonically) which meeting place to visit first, after which he strictly alternates his visits between them. At each place he looks at the noticeboard, and if it displays "here", he goes inside. If it does not display "here" it will display a number instead, in which case the tourist compares that number $K$ with the number $k$ on his notepad and does one of the following:

if $k<K-$ The tourist writes $K$ on his notepad (erasing $k$ ), and goes to the other place.

if $k>K-$ The tourist writes "here" on the noticeboard (erasing $K$ ), and goes inside.

if $k=K-$ The tourist chooses $K^{\prime}$, the next even number larger than $K$, and then flips a coin: if it comes up heads, he increases $K^{\prime}$ by a further one. He then writes $K^{\prime}$ on the noticeboard and on his notepad (erasing $k$ and $K$ ), and goes to the other place 2

A key characterisation of the Rabin's algorithm, which has also been proved elsewhere [8] is that, on termination all the tourists' will converge at the same meeting place, and that happens with probability 1. However it is not always the case that an "observer" can witness every state of the program that will lead to termination. For example, it is possible that the tourists will forever (according to an observer) keep updating their notepads and the noticeboards without deciding on a meeting place. This enforces an unbounded state behaviour on the algorithm. Nonetheless, given the unbounded state nature of the algorithm, our theoretical results still permit us to study a suitable performance attribute of the system: the expected number of rounds (or steps) of the protocol until termination (analogous to convergence).

\subsection{A pGCL snapshot of the Rabin's algorithm}

Fig. 4 (on the next page) gives an overview of the Rabin's choice-coordination problem in the pGCL. We call the two meeting places "left" and "right" as we discuss it and refer to the notations 3 accordingly. Bag lout (rout) is the bag of numbers held by tourists waiting to look at the left (right) noticeboard; bag lin ( rin) is the bag of numbers held by tourists who have already decided on the left (right) alternative; number $L(R)$ is the number on the left (right) noticeboard. Initially there are $A(B)$ tourists on the left (right), all holding the number zero; no tourist has yet made a decision, and both noticeboards show zero.

Execution is as follows: if some tourists are still undecided (so that lout (rout) is not yet empty), select one: the number he holds is $l(r)$. If some tourist has already decided on this alternative (so that lin (rin) is not empty), this tourist does the same; otherwise any of the three possibilities discussed above is executed.

\subsection{Computing average performance of the Rabin's algorithm}

In this section we discuss properties of the Rabin's algorithm sufficient for an analysis of its average performance. Since the unbounded state nature of the algorithm limits the scope of model checking on the

\footnotetext{
${ }^{2}$ For example if $K$ is 2 or 3 , then $K^{\prime}$ becomes 4 and then possibly 5 .

${ }^{3}[[\ldots]]$ — bag (multiset) brackets; $\square$-empty bag; $[[n]]^{N}$-bag containing $N$ copies all of value $n$; take $n$ from $b-$ a program command which chooses an element demonically from non-empty bag $b$, assigns it to $n$ and removes it from $b$; add $n$ to $b$-add element $n$ to bag $b ; \bar{n}$ - the "conjugate" of $n$, it is $n+1$ if $n$ is even and $n-1$ if $n$ is odd; \#b - the number of elements in a bag $b$.
} 


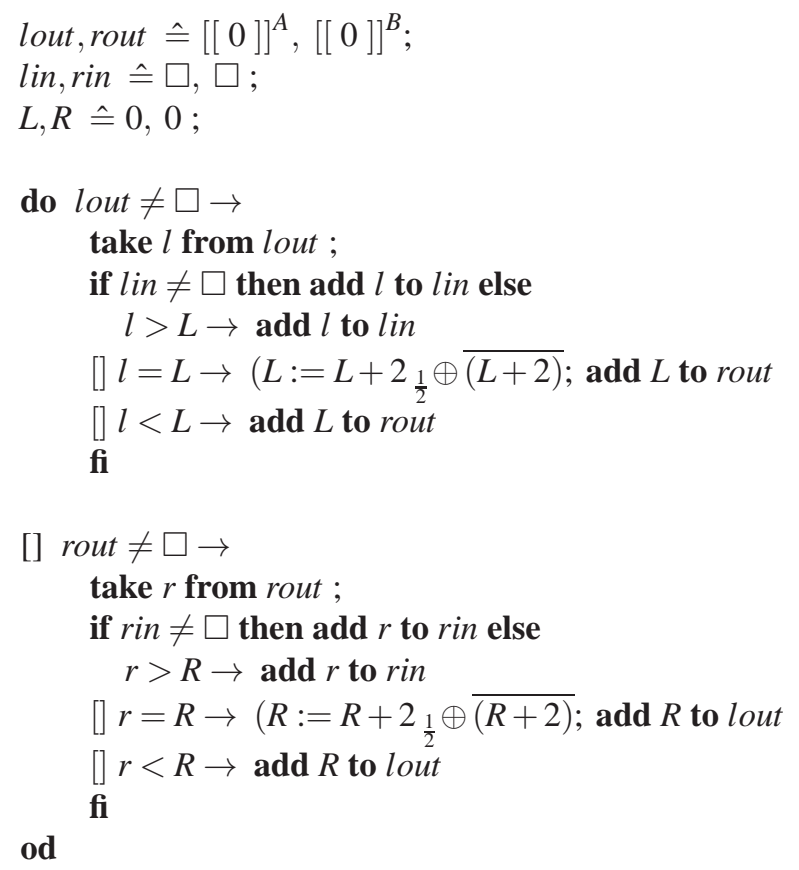

Figure 4: The Rabin's choice coordination algorithm in the pGCL (adapted from [8]).

original system, we must therefore compute a suitable abstraction prior to performance analysis. Nevertheless, with our proposed technique, it is possible to defeat the overhead incurred by model checking the unbounded state system just by model checking its information-preserving abstraction.

One performance property of interest is captured by computing the minimum probability Pmin, that within a finite number of steps $T$, the tourists eventually converge at the same meeting place on termination. In the logic PCTL [6], directly supported by the PRISM tool, we express this property as

$$
\text { Pmin }=?\left[\text { true } U^{\leq T}(\# \text { lin }=N) \mid(\# \text { rin }=N)\right],
$$

where $N$ represents the total number of tourists who will initially decide on where to meet i.e. $N=A+B$.

Similarly, with the reward structures [12] of the PCTL we compute the expected number of rounds of the protocol until termination. Again, this will be done on the protocol's abstraction using the specification:

$$
\text { Rmin } \mid \text { Rmax }=?[F(\# l i n=N) \mid(\# \operatorname{rin}=N)] .
$$

The parameters Rmin and Rmax respectively represent the expected minimum and maximum rewards (expected number of rounds) until the tourists eventually converge at the same meeting place. We note that states where the tourists have not yet met the convergence condition are worth a reward value of one.

In the sections that follow, we explain how we identify essential behaviours of the algorithm that will permit the construction of an information-preserving abstraction upon which the analysis can be performed.

\subsection{An information-preserving abstraction}

As earlier stated, even though the Rabin's algorithm has unbounded state behaviour, it is not data independent since the probabilistic update increments the variables $L, R$ etc. However there is still an 


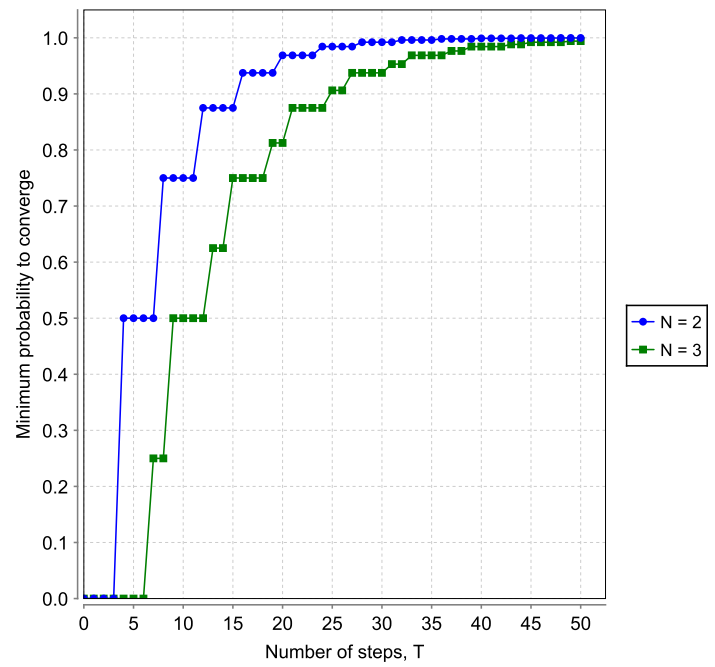

Figure 5: Performance result of Rabin's algorithm for $\mathrm{N}=2,3$.

\begin{tabular}{|c|c|c|}
\hline Number of Tourists & Rmin & Rmax \\
\hline $\mathbf{N}=\mathbf{2}$ & 2 & 7 \\
\hline $\mathbf{N}=\mathbf{3}$ & 2 & 11 \\
\hline
\end{tabular}

Figure 6: Expected number of steps

information-preserving abstraction, which we will now describe.

Observe that although the noticeboard values are incremented, they always maintain $|L-R| \leq 2$. In terms of the algorithm, the only information that needs to be preserved is the value $L-R$ and whether $L, R$ are odd or even. Finally, the relative values of the tourists' numbers to $L$ and $R$ also need to be recorded, as well as their location. This generates an information-preserving abstraction. In practice, we characterise the relationship between the noticeboard values using a fresh variable we call slot, which can only take values in $\{0,1,2\}$ - since the noticeboard values and hence the notepad values can only lie in one of these slots for any given state of the system. We define the slot variable as follows and interpret transitions in the abstract state with respect to the slot values:

$$
\text { slot } \hat{=}\left\{\begin{array}{lll}
0 & \text { if } \quad L=R \\
1 & \text { if } \quad L=R-2 \vee R=L-2 \\
2 & \text { if } \quad L=\bar{R} .
\end{array}\right.
$$

In the section that follows, we explain the performance results derived from the information-preserving abstraction. The results nevertheless give a precise summary of the performance of the original system.

\subsection{Experimental results}

We model the abstract behaviour discussed above for the base cases of even and odd number of tourists $(N=2,3)$ in the PRISM language, and similarly analyse the performance results as captured by the properties in (5) and (6), using the experimentation facility of the tool. A similar model construction and analysis for larger values of $N$ is also possible by repeating the same technique although very laborious. 
Fig. 5 captures the performance characteristics of the information-preserving abstraction of the Rabin's algorithm. It clearly establishes the termination property of the unbounded state system using just its abstraction: note that both graphs converge to probability 1 . In the original unbounded state system, achieving this is practically impossible. See the original model in the compendium of case studies at [1].

We also observe (Fig. 6) that the expected minimum and maximum number of rounds until termination can be model checked, and hence nevertheless gives an exact bound on the number of steps required for the unbounded state system to terminate. Again, in the unbounded state system, the result of computing Rmax for example is infinity, which in the PRISM tool is interpreted to mean that it is not possible for a terminating (or convergence) condition to be reached.

\section{Discussion}

While some probabilistic program logics allow programs to be compared even at abstract levels, for example using the techniques in [5, 3], the underlying logic of the pGCL supports the notion of program refinement and hence compositionality. This makes it easy to relate refinement over concrete states to their abstract counterparts and furthermore with the other probabilistic program logics, given any context.

Other approaches seek to use variations of counterexample guided predicate abstraction [10, 7] to automate finding sets of predicates which generate finer abstractions. One way to see the relationship with our approach would be to note that when an abstraction is observed to be information-preserving (according to Lem. 3 for example) then further refinement is unnecessary. Kwiatkowska et al. [11] propose an approach to estimate the accuracy of the analysis implied by any abstraction, confirming that for information-preserving abstractions the analysis is exact.

On the application level, one way to see the usefulness of our technique is in the recent research direction of linking proof-based verification with model checking for probabilistic systems [18, 19]. Since proof-based verification can cope with proofs over infinite state systems, a key challenge with this technique is then the identification and constructing of information-preserving abstractions upon which a model checking algorithmic verification can be performed. This is still an open problem.

\section{Conclusion and future work}

In this paper we have developed the theory of predicate abstraction for probabilistic programs within the framework of expectation transformers. We have similarly established a criterion to help discover when abstractions do not lose information especially for probabilistic programs; and we have demonstrated the applicability of the results to data independent programs (or at least their approximations).

Whilst our theoretical approach identifies when a set of predicates is information-preserving, it does not provide assistance for finding one. Even though we have computed the abstraction by hand, we quickly remark that applying the manual construction technique for $N>3$ would seem a laborious task. Note that our technique results in a huge success for verifying the termination condition of the algorithm when compared with the concrete system as modeled in the compendium of case studies at the URL [1].

However, a future direction for this work would be to develop an automated strategy which would construct abstractions "on the fly", given that our theoretical framework is rich enough to provide intuitions to identifying sets of suitable predicates to aid the construction of information-preserving abstractions.

Acknowledgement: The authors are grateful to the anonymous reviewers for their helpful comments. 


\section{References}

[1] PRISM: Probabilistic Symbolic Model Checker. URL: http: //www. prismmodelchecker. org/.

[2] Tarski A. A Lattice-theoretic Fixpoint Theorem and its Applications. Pacific Journal of Mathematics, 5:285309, 1955.

[3] Jou C. C. and Smolka S.A. Equivalences, Congruences, and Complete Axiomatizations for Probabilistic Processes. In J. Baeten and J. Klop, Editors, CONCUR 90 1st Int. Conf. on Concurrency Theory, Number 458 in LNCS Vol. 94:1-28, Springer, 1990.

[4] Fudenberg D. and E. Maskin. The Folk Theorem in Repeated Games with Discounting or with Incomplete Information. Econometrica, 1986.

[5] Larsen K. G. and Skou A. Bisimulation Through Probablistic Testing. Information and Computation, 94:128, 1991.

[6] Hansson H. and Jonsson B. A Logic for Reasoning about Time and Reliability. In Formal Aspects of Computing, 6(5):512-535, 1994.

[7] Hermanns H., Wachter B., and Zhang L. Probabilistic CEGAR. In Proc. of the 20th international Conference on Computer Aided Verification, Princeton, NJ, USA, 2008.

[8] McIver A. K. and Morgan C. C. Abstraction, Refinement and Proof for Probabilistic Systems. Monographs in Computer Science, Springer, Verlag, 2004.

[9] Clarke E. M., Grumberg O., and Peled D. A. Model Checking. MIT Press, 1999.

[10] Kattenbelt M., Kwiatkowska M., Norman G., and Parker D. Abstraction Refinement for Probabilistic Software. In Proc. of 10th International Conference on Verification, Model Checking and Abstract Interpretation (VMCAI ‘09), Springer, 2009.

[11] Kwiatkowska M., Norman G., and Parker D. Game-based Abstraction for Markov Decision Processes. In Proc. of 3rd International Conference on Quantitative Evaluation of Systems (QEST'06), pages 157-166, IEEE CS Press, 2006.

[12] Kwiatkowska M., Norman G., and Parker D. Stochastic Model Checking. In Proc. of SFM'07 vol. 4486 LNCS, 220-270, Springer, 2007.

[13] Rabin M. O. The Choice Coordination Problem. Acta Informatica vol. 17, 121 - 134, 1982.

[14] Wolper P. Expressing Interesting Properties of Programs in Propositional Temporal Logic. In Proc. of the 13th Annual Symposium on Principles of Programming Languages, pp. 184 - 193, ACM, 1986.

[15] Grimmett G. R. and Welsh D. Probability: An Introduction. Oxford Science Publications, 1986.

[16] Lazić R. A Semantic Study of Data Independence with Applications to Model Checking. DPhil Thesis, Oxford University Computing Laboratory, 1999.

[17] Ball T. Formalizing Counterexample-driven Refinement with Weakest Preconditions. Technical Report MSRTR-2004-134, Microsoft Research, Redmond, WA 98052, USA, 2004.

[18] Ndukwu U. Quantitative Safety: Linking Proof-based Verification with Model Checking for Probabilistic Systems. In Proc. First International Workshop on Quantitative Formal Methods (QFM 2009), Eindhoven, Netherlands, 2009.

[19] Ndukwu U. Generating Counterexamples for Quantitative Safety Specifications in Probabilistic B. Submitted to the Journal of Logic and Algebraic Programming (JLAP) URL: http://web.science.mq.edu.au/ ukndukwu/counterexamples.pdf, 2010.

[20] Dijkstra E. W. A Discipline of Programming. Prentice Hall International, Englewood Cliffs, N.J., 1976. 